\title{
Foreword
}

\section{Sport-Related Concussion: Heads Up!}

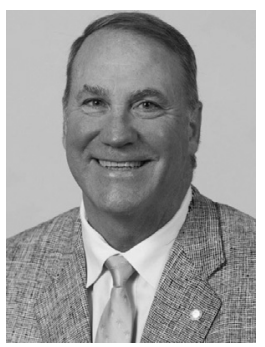

Mark D. Miller, MD Consulting Editor

There has been a lot of progress since the last time we covered this topic in Clinics in Sports Medicine a decade ago, including new research, an emphasis on no return to play the same day, and a better understanding of chronic traumatic encephalopathy. Nevertheless, there is still a great deal that we don't know. Thank you to Dr Kriz for putting together this treatise outlining the current state-of-the-art on sport-related concussion (SRC). This issue covers the entirety of this topic, beginning with the basics and bringing in both research and clinical management. The editor addresses all populations, including females, kids, and athletes, at all levels. But he didn't stop there, prevention, long-term effects, and future directions are all covered as well. In sum, this is an excellent update on SRC that I encourage all to read and incorporate into their sideline game bag!

Mark D. Miller, MD

Division of Sports Medicine Department of Orthopaedic Surgery

University of Virginia

James Madison University 400 Ray C. Hunt Drive, Suite 330 Charlottesville, VA 22908-0159, USA

E-mail address:

MDM3P@hscmail.mcc.virginia.edu 